\title{
Clinical performance of the Solana® Point-of-Care Trichomonas Assay from clinician-collected vaginal swabs and urine specimens from symptomatic and asymptomatic women
}

\author{
CA Gaydos ${ }^{1}$, J Schwebke ${ }^{2}, \mathbf{J ~ D o m b r o w s k i ~}^{3}, \mathbf{J ~ M a r r a z z o}^{2,3}$, J Coleman $^{1}$, B Silver ${ }^{1}, \mathbf{M}$ \\ Barnes $^{1}$, L Crane ${ }^{4}$, and P Fine ${ }^{4}$ \\ ${ }^{1}$ Johns Hopkins University School of Medicine, Baltimore Maryland ${ }^{2}$ University of Alabama at \\ Birmingham School of Medicine, Birmingham Alabama ${ }^{3}$ University of Washington School of \\ Medicine ${ }^{4}$ Planned Parenthood, Houston, Texas
}

\begin{abstract}
Background-Solana ${ }^{\circledR}$ (Quidel) is a new rapid ( $<40 \mathrm{~min}$.) point-of-care (POC) test for qualitative detection of Trichomonas vaginalis (TV) DNA. The assay has two steps: 1) specimen preparation, and 2) amplification and detection using isothermal Helicase-Dependent Amplification (HDA). The objective was to demonstrate the performance of Solana for vaginal swabs and female urines based on comparison to wet mount and TV culture. Performance was also compared to the Aptima-TV assay.

Methods-Urine and four clinician-collected vaginal swabs were collected. The first two were used for FDA composite reference (wet mount; InPouch TV Culture). The third swab was used for Solana. Sensitivity/specificity were based on the reference method. A specimen was considered positive if either test was positive. The fourth swab was for Aptima-TV.

Results - Vaginal swabs and urines were obtained from 501 asymptomatic and 543 symptomatic women. Prevalence of TV by was $11.5 \%$. For swabs, Solana ${ }^{\circledR}$ demonstrated high sensitivity and specificity from asymptomatic (100\%/98.9\%) and symptomatic (98.6\%/98.5\%) women, as well as for urines from asymptomatic (98.0\%/98.4\%) and symptomatic (92.9\%/97.9\%) women, compared to the reference method. Compared to Aptima-TV, the sensitivity/specificity was $89.7 \% / 99.0 \%$ for swabs and $100 \% / 98.9 \%$ for urines.
\end{abstract}

Conclusions-The Solana ${ }^{\circledR}$ assay performed well compared to the reference assays.

\section{Keywords}

Trichomonas vaginalis; trichomoniasis; Point-of-Care test; Solana

Corresponding Author: Charlotte A. Gaydos, MS, MPH, DrPH, 855 N Wolfe St, 530 Rangos Building, Baltimore, MD, 21205, cgaydos@jhmi.edu; 410-614-0932; fax 410-614-9775.

Declaration of Interest

The authors have no relevant affiliations or financial involvement with any organization or entity with a financial interest in or financial conflict with the subject matter or materials discussed in the manuscript. This includes employment, consultancies, honoraria, stock ownership or options, expert testimony, grants or patents received or pending, or royalties. 


\section{Introduction}

Trichomonas vaginalis (TV), a motile protozoan parasite, causes the most prevalent nonviral sexually transmitted infection (STI) in the world. It is estimated to infect 3.7 million people in the United States. ${ }^{1}$ The World Health Organization estimates that 276.4 million new cases of trichomonas per year in adults between the ages of 15 and 49 years in $2008 .^{2}$ These infections represent the most common curable STI in young, sexually active men and women. ${ }^{2}$

Trichomonas infections in women have been associated with poor reproductive outcomes such as low birth weight (LBW) and premature birth. ${ }^{3-5}$ There was a reported attributable risk of trichomonas associated with LBW in Blacks of $11 \%$ vs. $1.6 \%$ in Hispanics, and $1.5 \%$ in Whites in a cohort of over 13,000 women. ${ }^{3}$ The National Health and Nutrition Examination Survey (NHANES) 2001-2004 estimated that 3.1\% of women in the United States have TV. ${ }^{6}$ TV has been associated with other STIs among women in the U.S. population and importantly over $80 \%$ of cases were asymptomatic. ${ }^{7}$ T. vaginalis infection has been also associated with two to three-fold significant increased risks of HIV acquisition, and pelvic inflammatory disease (PID) among HIV-infected women. ${ }^{8-11}$ Older age and health disparities are also significant epidemiological factors in trichomonas infections which have been reported to affect over $11 \%$ of women age $\geq 40$ years and $13 \%$ of black women in the United States. ${ }^{12,13}$ Because of such sequelae and the asymptomatic nature of $T$. vaginalis infections, it is important to test women for these infections.

The conventional methods to detect $T$. vaginalis in vaginal swabs are wet mount/saline microscopy and culture techniques, but both are insensitive (40-60\%) compared to newer nucleic acid amplification tests (NAATs). ${ }^{4,12,14-17}$ The OSOM test is a rapid point of care (POC) test that is CLIA waived and more sensitive than either wet preparation and culture sensitivities with reported sensitives of $>80 \%$ compared to NAAT assays ( $>90 \%)$, making it ideal for POC testng. ${ }^{14,17}$ NAAT assays have been shown to be the most sensitive but are not CLIA waived or POC yet. ${ }^{12,14-17}$ The objective of this study was to demonstrate the performance of the new rapid Solana ${ }^{\circledR}$ TV-Assay (Quidel Corporation, Athens Ohio) for clinician-collected vaginal swabs and female urines in a multi-center study, based on comparison of Solana results to those by wet mount and TV culture. A secondary objective was to determine performance compared to the Aptima-TV assay.

\section{Methods}

Symptomatic women and asymptomatic women who were 14 years or older were enrolled from high and low prevalence clinics from five geographically diverse areas. The study was performed January to March 2016 at four locations in the United States. Specimens were obtained from each subject after written informed consent was obtained. Inclusion criteria included women who presented with symptoms of $T$. vaginalis and asymptomatic women who were scheduled for a pelvic examination or screening for $T$. vaginalis. Patient age or date of birth was obtained. 
The study was conducted in accord with the Health Insurance Portability and Accountability Act (HIPAA) and approved by the associated Institutional Review Boards.

For each subject, 4 vaginal swab specimens were clinician-collected and were used for reference (wet mount and culture (InPouch ${ }^{\mathrm{TM}}$ TV, BioMed Diagnostics, Inc., White City, Oregon).), Solana ${ }^{\circledR}$, and Aptima-TV (Gen-Probe/Hologic, San Diego, CA) testing. The first two swabs were used for saline wet mount, read by the clinician, and TV culture. The 3rd polyester swab was used for Solana Assay testing. The 4th was used for Aptima-TV. A firstcatch urine was collected from each subject for testing by all 4 methods. All sensitivity/ specificity calculations were initially based on a FDA-composite reference method (saline wet mount and InPouch-TV-culture). A specimen was considered positive if either test was positive. For samples that were positive by Solana ${ }^{\circledR}$ and negative by saline wet mount and culture, the Aptima TV was used as a confirmation of a true positive.

The Solana ${ }^{\circledR}$ Trichomonas Assay is an in vitro qualitative nucleic acid amplification test for the detection of $T$. vaginalis to aid in the diagnosis of trichomoniasis using the HelicaseDependent Amplification (HDA) technology and the Solana ${ }^{\circledR}$ instrument (Figure 1). To detect $T$. vaginalis directly from trichomoniasis-suspected specimens, the assay targets a conserved repeat sequence of the T. vaginalis genome. The assay consists of two major steps: 1) specimen preparation, and 2) amplification and detection of target sequence specific to T. vaginalis DNA using isothermal Helicase-Dependent Amplification (HDA) in the presence of target-specific fluorescence probe. The assay is intended for use in clinical laboratories as an aid in the diagnosis of trichomoniasis.

\section{Data Analysis}

Descriptive statistical data analyses were performed to summarize the data in numbers and percentages. We also used the point estimate and its corresponding confidence intervals to determine the statistical significance. P-values of $<0.05$ were considered significant. SAS version 9.4 (SAS Institute, Cary, North Carolina, USA) was used for all statistical analyses.

\section{Results}

Clinician-collected vaginal swabs and urines $(\mathrm{N}=1044)$ were obtained from 501 asymptomatic and 543 symptomatic women (Table 1). The prevalence of TV by swabs and/or urines was $11.5 \%$. For clinician-collected swabs, the Solana Trichomonas Assay demonstrated high sensitivity and specificity from asymptomatic (100\%/98.7\%) and symptomatic $(98.6 \% / 98.5 \%)$ women, as well as for urines from asymptomatic $(98.0 \% /$ 98.4\%) and symptomatic (92.9\%/97.9\%) women, when compared to the FDA-composite reference method (Table 2). Compared to Aptima-TV, the sensitivity/specificity performance was $89.7 \% / 99.0 \%$ for swabs and $100 \% / 98.9 \%$ for urines (Table 3 ).

\section{Discussion}

The Solana ${ }^{\circledR}$ TV Assay demonstrated high sensitivity and specificity, regardless of symptoms, compared to the routinely used for standard of care tests of saline wet preparation microscopy and culture. Additionally, this assay also showed high agreement 
compared to a NAAT assay with an overall agreement of $97.8 \%$. There are now two FDA cleared NAAT assays for trichomonas ${ }^{12,18}$, and it is generally accepted that NAATs for TV are more sensitive than culture. ${ }^{15}$ However, the reference standard set by the FDA for this study was saline wet preparation microscopy and culture, which was the composite positive comparator (CPC). Thus, the study design and the CPC used may have overestimated the point estimates for this trial, since NAATs have been demonstrated to be more sensitive than culture.

The rapid Solana ${ }^{\circledR}$ TV Assay appeared to perform better than saline microscopy/culture by detecting more positive results than the defined CPC. However, it did not detect more positives than the NAAT.

There are limitations to the interpretation of our study in that the Solana appeared to detect more positives than the CPC. There may have been lower number of positives in the CPC assays due to delays in incubating cultures and reading saline microscopy tests, transportation delays or variability in interpretation of the results of the comparator assays, both of which are subjective in nature. The possibility of false positives by the NAAT test also cannot be excluded, but they are unlikely since the platform is a closed, robotic platform, which should lower the likelihood of cross contamination by a laboratory.

The Solana ${ }^{\circledR}$ TV Assay provided evidence that an amplified molecular assay can perform as well as saline microscopy/culture and provided comparable results as another FDA cleared NAAT assay for detection of trichomoniasis in symptomatic and asymptomatic women, while yielding results in 45 minutes. Although the requirement of equipment and a time of 45 min are not attributes of a perfect POC test, but this platform can still be useful in many clinical situations, but its usefulness in remote clinics without trained personnel and in resource-poor settings will need to be further evaluated. It is now FDA cleared as moderately complex, but its potential to be CLIA waived in the future may increase its usefulness as a POC test that can be used for treatment of patients in remote areas before they leave the clinic. It appeared to yield a similar or slightly better sensitivity in this study to another rapid, commercially available unamplified antigen trichomonas assay. ${ }^{14}$

Because of the data accumulating for the association of trichomonas infections with adverse events, such as HIV transmission and perinatal morbidity, it seems judicious to promote public health recognition for more screening for trichomonas, especially since modeling studies demonstrate the potential to improve health outcomes. ${ }^{11,18-22}$ Interestingly, recent research linking trichomonas infections to prostate cancer in men has been accumulating and may add reasons to be more actively screen men for trichomonas. ${ }^{23-24}$ The availability of serological detection of circulating antibody to trichomonas may be an additional epidemiological tool for diagnosis of chronic infections, when only very limited amount of pathogens are released in urine or in vaginal secretions. ${ }^{25}$

In summary, the rapid Solana ${ }^{\circledR}$ TV Assay showed a high sensitivity and specificity in both asymptomatic and symptomatic women in 45 minutes of test time, when compared to the composite reference method that included saline microscopy and InPouch TV culture. 


\section{Acknowledgments}

Funding: The manuscript was funded by Quidel Corporation, Athens, Ohio, U54EB007958, NIBIB, NIH; U-01068613, NIH, NIAID

\section{References}

1. Satterwhite CL, Torrone E, Meites E, et al. Sexually Transmitted Infections Among US Women and Men: Prevalence and Incidence Estimates, 2008. Sex Transmit Dis. 2013; 40:187-93.

2. World Health Organization. Global Incidence and prevalence of selected curable sexually transmitted infections-2008. WHO; Geneva: 2012. accessed 09-28-15:1-20

3. Cotch MF, Pastorek JG, Nugent RP, et al. Trichomonas vaginalis associated with low birth weight and preterm delivery. Sex Transmit Dis. 1997; 24:353-60.

4. Schwebke JR, Burgess D. Trichomoniasis. Clin Microbiol Review. 2004; 17:794-803.

5. Silver BJ, Guy RJ, Kaldoe JM, Jamil MS, Rumbold AR. Trichomonas vaginalis as a cause of perinatal morbidity: A systematic review and meta-analysis. Sex Transmit Dis. 2014; 41:369-76.

6. Sutton M, Sternberg M, EH K, McQuillan G, Berman S, Markowitz L. The prevalence of Trichomonas vaginalis infection among reproductive-age women in the United States, 2001-2004. Clin Inf Dis. 2007; 45:1319-26.

7. Allsworth JE, Ratner JA, Peipert JF. Trichomonas and other sexually transmitted infections: Results from the 2001-2004 National Health and Nutrition Examination Surveys. Sex Transmit Dis. 2009; 36:738-44.

8. Van Der Pol B, Kwok C, Pierre-Louis B, et al. Trichomonas vaginalis Infection and Human Immunodeficiency Virus Acquisition in African Women. J Infect Dis. 2008; 197:548-54. [PubMed: 18275275]

9. Moodley P, Wilkinson D, Connolly C, Moodley J, Sturm AW. Trichomonas vaginalis is associated with pelvic inflammatory disease in women infected with Human Immunodeficiency Virus. Clin Infect Dis. 2002; 34:519-22. [PubMed: 11797180]

10. Hughes J, Baeten J, Lingappa J, et al. Determinants of per-coital act HIV-1 infectivity among African HIV-1-serodiscordant couples. J Infect Dis. 2012; 205:358-65. [PubMed: 22241800]

11. Kissinger P, Adamski A. Trichomoniasis and HIV interactions: a review. Sex Transmit. 2013; 89:426-33.

12. Schwebke JR, Hobbs M, Taylor S, et al. Molecular testing for Trichomonas vaginalis in women: Results of a pivotal US clinical trial. J Clin Microbiol. 2011; 49:4106-11. [PubMed: 21940475]

13. Ginocchio CC, Chapin K, Smith JS, et al. Prevalence of Trichomonas vaginalis and coinfection with Chlamydia trachomatis and Neisseria gonorrhoeae in the United States as determined by the Aptima Trichomonas vaginalis nucleic acid amplification assay. J Clin Microbiol. 2012; 50:26018. [PubMed: 22622447]

14. Huppert JS, Mortensen JE, Reed JL, et al. Rapid Antigen Testing Compares Favorably with Transcription-Mediated Amplification Assay for the Detection of Trichomonas vaginalis in Young Women. Clin Infect Dis. 2007; 45:194-8. [PubMed: 17578778]

15. Nye MB, Schwebke JR, Body BA. Comparison of APTIMA Trichomonas vaginalis transcriptionmediated amplification to wet mount microscopy, culture, and polymerase chain reaction for diagnosis of trichomoniasis in men and women. Am J Obstet Gyneco. 2009; 200:188.e1-e7.

16. Van Der Pol B, Williams JA, Taylor SN, et al. Detection of Trichomonas vaginalis DNA by Use of Self-Obtained Vaginal Swabs with the BD ProbeTec Qx Assay on the BD Viper System. J Clin Microbiol. 2014; 52:885-889. [PubMed: 24391200]

17. Van Der Pol. Clinical and laboratory testing for Trichomonas vaginalis infection. J Clin Microbiol. 2016; 54:7-12. [PubMed: 26491181]

18. Anderson BL, Firnhaber C, Liu T, et al. Effect of trichomoniasis therapy on genital HIV burden among African women. Sex Transm Dis. 2012; 39:638-642. [PubMed: 22797689]

19. Chesson HW, Blandford JM, Pinkerton SD. Estimates of the annual number and cost of new HIV infections among women attributable to trichomoniasis in the United States. Sex Transm Dis. 2004; 31:547-551. [PubMed: 15480116] 
20. Kissinger P, Amedee A, Clark RA, et al. Trichomonas vaginalis treatment reduces vaginal HIV-1 shedding. Sex Transm Dis. 2009; 36:11-16. 2009. [PubMed: 19008776]

21. Quinlivan EB, Patel SN, Grodensky CA, Golin CE, Tien HC, Hobbs MM. Modeling the impact of Trichomonas vaginalis on HIV transmission in HIV-infected individuals in medical care. Sex Transm Dis. 2012; 39:671-677. [PubMed: 22902662]

22. Trintis J, Epie N, Boss R, Riedel S. Neonatal Trichomonas vaginalis infection: a case report and review of literature. International Journal of STD \& AIDS. 2010; 21:606-607. [PubMed: 20975098]

23. Stark JR, Judson G, Alderete JF, Mundodi V, Kucknoor AS, Giovannucci EL, Platz EA, Sutcliffe S, Fall K, Kurth T, Ma J, Stampfer MJ, Mucci LA. Prospective study of Trichomonas vaginalis infection and prostate cancer incidence and mortality: Physicians' Health Study. J Natl Cancer Inst. 2009 Oct 21; 101(20):1406-11. DOI: 10.1093/jnci/djp306 [PubMed: 19741211]

24. Twu O, Dessí D, Vu A, Mercer F, Stevens GC, de Miguel N, Rappelli P, Cocco AR, Clubb RT, Fiori PL, Johnson PJ. Trichomonas vaginalis homolog of macrophage migration inhibitory factor induces prostate cell growth, invasiveness, and inflammatory responses. Proc Natl Acad Sci U S A. 2014 Jun 3; 111(22):8179-84. DOI: 10.1073/pnas.1321884111 [PubMed: 24843155]

25. Ton Nu PA, Rappelli P, Dessì D, Nguyen VQ, Fiori PL. Kinetics of circulating antibody response to Trichomonas vaginalis: clinical and diagnostic implications. Sex Transm Infect. 2015 Dec; 91(8):561-3. [PubMed: 25887282] 


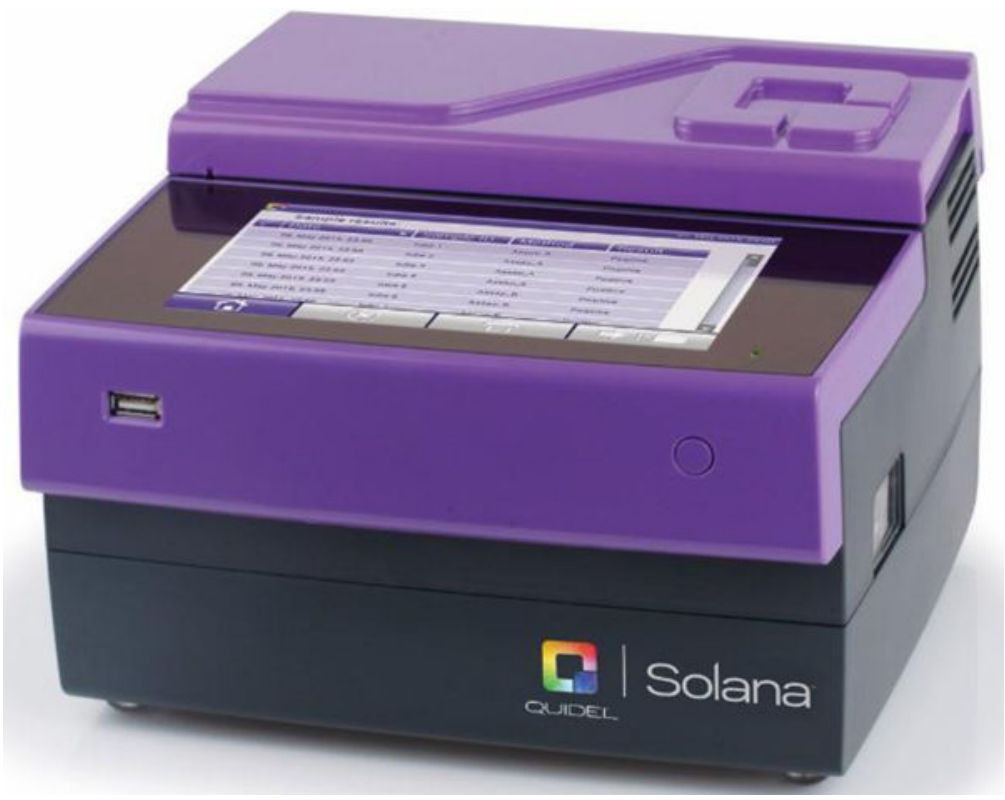

Figure 1.

Solana Platform. 


\section{Table 1}

Characteristics of women enrolled in the Solana study for Trichomonas vaginalis

\begin{tabular}{ccccc}
\hline Characteristics & Category & $\begin{array}{c}\text { Combined (\%) } \\
\mathbf{N = 1 0 4 4}\end{array}$ & $\begin{array}{c}\text { Symptomatic (\%) } \\
\mathbf{N = 5 4 3}\end{array}$ & $\begin{array}{c}\text { Asymptomatic (\%) } \\
\text { N=501 }\end{array}$ \\
\hline Age (years) & $16-24$ & $33.0 \%(344 / 1044)$ & $37.4 \%(203 / 543)$ & $28.1 \%(141 / 501)$ \\
& $25-29$ & $23.6 \%(246 / 1044)$ & $25.4 \%(138 / 543)$ & $21.6 \%(108 / 501)$ \\
& $30-34$ & $11.8 \%(123 / 1044)$ & $12.2 \%(66 / 543)$ & $11.4 \%(57 / 501)$ \\
& $\geq 35$ & $31.7 \%(331 / 1044)$ & $25.0 \%(136 / 543)$ & $38.9 \%(195)$
\end{tabular}




\section{Table 2}

Sensitivity and Specificity of the Solana Assay for Trichomonas vaginalis compared to the Composite Reference Methods of Saline Wet Mount and Culture

\begin{tabular}{lllll} 
Vaginal Swabs & Sensitivity & \%CI 95 & Specificity & \% CI 95 \\
\hline Asymptomatic women & $100 \%$ & $92.7 \%$ to $100 \%$ & $98.7 \%$ & $97.1 \%$ to $99.4 \%$ \\
Symptomatic women & $98.6 \%$ & $92.3 \%$ to $99.7 \%$ & $98.5 \%$ & $97.0 \%$ to $99.3 \%$ \\
Total & $99.2 \%$ & $95.4 \%$ to $99.9 \%$ & $98.6 \%$ & $97.6 \%$ to $99.2 \%$ \\
Urine & & & & \\
\hline Asymptomatic women & $98.0 \%$ & $89.5 \%$ to $99.6 \%$ & $98.4 \%$ & $96.8 \%$ to $99.2 \%$ \\
Symptomatic women & $92.9 \%$ & $84.3 \%$ to $96.9 \%$ & $97.9 \%$ & $96.2 \%$ to $98.8 \%$ \\
Total & $95.0 \%$ & $89.5 \%$ to $97.7 \%$ & $98.2 \%$ & $97.1 \%$ to $98.8 \%$ \\
\hline
\end{tabular}


Table 3

Sensitivity and Specificity of the Solana Assay for Trichomonas vaginalis compared to the Aptima-TV

\begin{tabular}{lllll} 
Vaginal Swabs & Sensitivity & \%CI 95 & Specificity & \%CI 95 \\
\hline Combined & $89.7 \%$ & $83.5 \%$ to $93.89 \%$ & $99.0 \%$ & $98.1 \%$ to $99.5 \%$ \\
Urine & & & & \\
\hline Combined & $100 \%$ & $96.9 \%$ to $100 \%$ & $98.9 \%$ & $98.0 \%$ to $99.4 \%$ \\
\hline
\end{tabular}

